11,12

\title{
Кинетика преципитации в бинарных сплавах вблизи границ зерен
}

\author{
(C) П.Е. ЛЬвов ${ }^{1}$, В.В. Светухин ${ }^{1,2}$ \\ ${ }^{1}$ Ульяновский государственный университет, \\ Ульяновск, Россия \\ ${ }^{2}$ Институт нанотехнологий микроэлектроники РАН, \\ Москва, Россия \\ E-mail: LvovPE@sv.uven.ru, Ipe10@mail.ru
}

(Поступила в Редакцию 30 октября 2017 г.)

На основе метода функционала плотности свободной энергии рассмотрено влияние границ зерен на процесс преципитации в бинарных сплавах. Проведен сравнительный анализ кинетики преципитации для монокристаллического фрагмента твердого раствора, а также фрагмента, содержащего участок границы двух зерен. Установлено влияние границ зерен на кинетику среднего радиуса преципитатов, их концентрации, а также скорости зарождения для нескольких составов сплава.

Работа выполнена в рамках выполнения государственного задания Минобрнауки России (проект № 16.2773.2017.ПЧ)и проекта РФФИ - Правительство Ульяновской области № 16-42-732113.

DOI: $10.21883 /$ FTT.2018.04.45694.305

\section{1. Введение}

Подавляющее большинство твердых растворов на основе металлов являются поликристаллами, т.е. состоят из большого числа зерен, представляющих собой фрагменты с однородной кристаллической структурой [1-3]. Области сопряжения (границы) соседних зерен, имеющих различную ориентацию, представляют собой дефекты кристаллической структуры и могут приводить к изменению энергии взаимодействия между компонентами твердого раствора [4-6]. В результате границы зерен могут оказывать существенное влияние на различные характеристики материалов, например, на их прочностные свойства [2], распределение фаз [2,3], скорости диффузии компонентов [2,6-9] и др.

Целью данной работы является установление особенностей зарождения и роста выделений второй фазы (преципитатов) в двухкомпонентных твердых растворах с учетом влияния границ зерен. При этом должны быть установлены особенности кинетики таких важных характеристик распределения фаз, как средний размер, концентрация преципитатов, а также скорость зарождения. Рассмотрение кинетики распада твердого раствора предполагается провести на основе разработанной авторами феноменологической модели [10], базирующейся на методе функционала плотности свободной энергии [11-15], а также на предположении о различии параметров взаимодействия на границе и в объеме зерна. Данное приближение в рамках единого подхода позволило выявить наличие различных типов распределения компонентов между зернограничной областью и объемом зерна [10], такие как обогащение и обеднение зернограничной области атомами растворенного компонента, преимущественная преципитация вблизи границ зерен, конкурентная преципита- ция в объеме зерна и вблизи зернограничной области и др.

\section{2. Основные приближения и расчетные формулы}

Рассмотрим трехмерный фрагмент бинарного сплава, имеющего форму куба, ребро которого равно $L$. Пусть внутри куба расположена граница двух зерен, описываемая плоскостью $z=L / 2$.

Предположим, что как внутри рассматриваемых зерен, так и вблизи границы для сплава выполняется приближение регулярного раствора. Если считать, что атомы сорта $A$ соответствуют растворителю, атомы сорта $B$ - растворенному веществу, то энергия смешения в расчете на одну частицу $g \equiv g\left(c_{B}\right)$ может быть записана в виде

$$
g\left(c_{B}\right)=\Omega c_{A} c_{B}+k_{B} T\left[c_{B} \ln c_{B}+c_{A} \ln c_{A}\right],
$$

где $T$ - температура, $k_{B}$ - постоянная Больцмана, $c_{A}$ и $c_{B}$ - концентрация атомов растворителя и растворенного вещества соответственно $\left(c_{A}+c_{B}=1\right), \Omega-$ параметр квазихимического взаимодействия, зависящий от координат $\Omega=\Omega(x, y, z)[10]$.

Будем считать, что вдали от границы зерна параметр взаимодействия равен значению, определяемому для макроскопических монокристаллических фрагментов твердого раствора $\Omega_{0}$, а вблизи границы зерна изменяется и оказывается равным $\Omega_{\mathrm{GB}}$. Предположим, что энергия смешения компонентов раствора повышается по мере приближения к границе зерна $\left(\Omega_{\mathrm{GB}}>\Omega_{0}\right)$. Изменение параметра квазихимического взаимодействия $\Omega$ вблизи границ зерен может быть связано либо с дефор- 
мацией кристаллической решетки [12], либо с изменением энергии парного взаимодействия между компонентами раствора [4-6]. Как правило, считается, что параметры взаимодействия изменяются в достаточно узком слое, характерная толщина которого $\delta$ составляет несколько периодов решетки (см. например, [5,6]). В качестве модельной аппроксимации данной зависимости удобно использовать степенные или экспоненциальные функции координат [10], отражающие плавное изменение параметра взаимодействия от $\Omega_{0 д о} \Omega_{\mathrm{GB}}$ на характерной толщине зернограничного слоя $\delta$. В более общем случае, когда требуется рассматривать более двух зерен, а также учитывать движение самих границ (см. например, [16]), параметр квазихимического взаимодействия $\Omega$ может быть связан с одним или несколькими параметрами порядка, отражающими различную ориентацию зерен друг относительно друга.

В качестве модельной зависимости параметра взаимодействия от координат для описанной системы выберем экспоненциальную функцию вида

$$
\Omega(x, y, z)=\Omega_{0}\left(1+\Delta \exp \left[-\frac{|z-L / 2|}{\delta}\right]\right) .
$$

Здесь параметр $\Delta=\left(\Omega_{\mathrm{GB}}-\Omega_{0}\right) / \Omega_{0}$ определяет максимальное различие между параметрами взаимодействия. Знак и величина параметра $\Delta$ определяют особенности распределения компонентов раствора между зернограничной областью и объемом зерна [10].

Моделирование эволюции концентрационного поля в пересыщенном твердом растворе с учетом зависимости параметра взаимодействия $\Omega$ от координат может быть проведено на основе модифицированного уравнения Кана-Хилларда [10]

$$
\frac{\partial c_{B}}{\partial t}=\nabla\left[M \cdot \nabla\left(\frac{\partial g}{\partial c_{B}}-\nabla\left(\kappa \nabla c_{B}\right)\right)\right] .
$$

где $M-$ подвижность, $\kappa-$ коэффициент градиентной энергии, который связан с параметром квазихимического взаимодействия $\kappa=\kappa_{0} \Omega(x, y, z) a^{2}, a-$ период решетки, $\kappa_{0}-$ постоянная величина, зависящая от потенциала межатомного взаимодействия компонентов раствора $[11,12]$.

Считая подвижность $M$ постоянной величиной и вводя параметр порядка $\eta=c_{B}-c_{A}=2 c_{B}-1$, уравнение (3) может быть приведено к виду

$$
\frac{\partial \eta}{\partial \tau}=\nabla^{* 2}\left[\phi-\nabla^{*}\left(\kappa^{*} \nabla^{*} \eta\right)\right]
$$

где $\kappa^{*}=\frac{\kappa}{\Omega_{0} a^{2}}, \tau=t \frac{M \Omega_{0}}{a^{2}}, \mathbf{r}^{*}=\mathbf{r} / a, \nabla^{*}=a \nabla, a-$ период решетки, $\phi-$ безразмерная функция, определяемая как

$$
\phi \equiv-2 \Omega^{*} \eta+T^{*} \ln \left[\frac{1+\eta}{1-\eta}\right],
$$

где использованы обозначения $T^{*}=2 k_{B} T / \Omega_{0}, \Omega^{*}=$ $=\Omega(x, y, z) / \Omega_{0}$.
Если считать граничные условия периодическими, то решение уравнения (4) может быть проведено на основе спектрального метода $[17,18]$, использование которого приводит к разностной схеме [10]

$$
\begin{aligned}
\hat{\eta}_{\mathbf{k}}^{n+1}=\hat{\eta}_{k}^{n} & -\frac{\Delta \tau|\mathbf{k}|^{2}}{1+\Delta \tau \kappa_{0} \tilde{\Omega}|\mathbf{k}|^{4}} \\
& \times\left\{\hat{\phi}_{k}^{n}-i \mathbf{k} \mathscr{F}_{k}\left[\kappa_{0} \Omega^{*} F_{k^{\prime}}^{-1}\left[i \mathbf{k}^{\prime} \hat{\eta}_{k^{\prime}}^{n}\right]\right]\right\},
\end{aligned}
$$

где $\hat{\eta}_{\mathbf{k}}^{n+1}-$ Фурье-образ параметра порядка в момент времени $\tau_{n}=\Delta \tau \cdot n, \Delta \tau-$ шаг по времени, $\mathscr{F}_{k}[\cdot]$ и $\mathscr{F}_{k^{\prime}}^{-1}[\cdot]$ - прямое и обратное преобразования Фурье соответственно, $\hat{\phi}_{k}^{n}-$ Фурье-образ производной от плотности свободной энергии в момент времени $\tau_{n}, \mathbf{k}$ и $\mathbf{k}^{\prime}$ - волновые векторы, $\tilde{\Omega}-$ безразмерная константа, обеспечивающая устойчивость разностной схемы (5), которая была принята равной $\tilde{\Omega}=1$.

Начальное распределение параметра порядка $\eta\left(\mathbf{r}^{*}, \tau\right)$ определялось на основе распределения Гаусса, характеризуемого средним значением $\langle\eta\rangle=2 c_{M}-1\left(c_{M}-\right.$ исходный состав сплава) и дисперсией, вытекающей из теории Орнштейна-Цернике [13] (см. также [10,19-21])

$$
\sigma^{2}=v_{0} k_{B} T\left(\left.V \frac{\partial^{2} g}{\partial c^{2}}\right|_{c_{M}}\right)^{-1}
$$

где $v_{0}-$ объем, приходящийся на один атом.

\section{3. Основные характеристики распределения фаз и методика их расчета}

Основными величинами, характеризующими распределение фаз в бинарном сплаве являются: концентрация $X_{C}$ и средний размер преципитатов $\langle R\rangle$. Для расчета данных характеристик сначала выделялись узлы, соответствующие второй фазе. Ко второй фазе относились все узлы решетки, для которых усредненный состав по всем узлам в окрестности сферы $r=2 a$ превышал пороговое значение $C_{\min }=50$ at.\%. Идентификация преципитатов проводилась на основе метода ближайшего соседа [20-22], в соответствии с которым узлы, находящиеся на расстоянии меньше или равном $r$, относятся к одному и тому же преципитату, и к разным преципитатам, если расстояние между узлами превышает $r$.

Линейный размер преципитата характеризовался эквивалентным радиусом $R=\left(\frac{3 v_{0} N}{4 \pi}\right)^{1 / 3}[20,21]$, где $N-$ количество узлов решетки, отнесенных к рассматриваемому преципитату.

Полная концентрация преципитатов и их средний размер вычислялись с помощью формул: $X_{C}=\frac{\Sigma_{R} n_{R}}{(L-1)^{3}}$, $\langle R\rangle=\sum_{R} R n_{R} / \sum_{R} n_{R}$, где $n_{R}-$ количество преципитатов, имеющих размер $R$, обнаруженных в рассматриваемом фрагменте твердого раствора. 
Важнейшей характеристикой, используемой в теории фазовых переходов, является скорость зародышеобразования (скорость нуклеации), которая представляет собой количество зародышей, образующихся в единицу времени в единице объема [3]. Данная величина рассчитывалась как первая производная от концентрации преципитатов $X_{C}$ по времени $\tau: I_{C}=\frac{d X_{C}}{d \tau}$ (см. [3,21]). Расчет производной проводился с помощью трехточечной разностной схемы, обеспечивающей второй порядок точности.

Моделирование распада бинарных сплавов с помощью разностной схемы (5), а также анализ распределения фаз проводился с помощью видеокарты GTX 1080.

\section{4. Параметры моделирования}

Рассмотрим формирование выделений второй фазы вблизи границы зерна, которое реализуется, если параметр взаимодействия $\Omega$ увеличивается по мере приближения к границе [10]. В целях определения влияния границ зерен на кинетику распада твердых растворов проведем сравнительный анализ для сплавов, характеризуемых параметрами $\Delta=0$ и $\Delta=0.5$. Первый случай $(\Delta=0)$ соответствует монокристаллу, т.е. однородному сплаву без зернограничной области. Второй случай соответствует повышению параметра квазихимического взаимодействия $\Omega$ по мере приближения к границе зерна.

Для каждого из рассматриваемых параметров $\Delta$ проведем расчет распада бинарных сплавов, имеющих составы $c_{M}=12,14,16$ at.\% при температуре $T^{*}=0.65$. Коэффициент $\kappa_{0}$ примем равным $\kappa_{0}=0.2$. Характерную ширину зернограничного слоя $\delta$ будем считать равной $\delta=2 a$.

Моделирование проводилось для трехмерных фрагментов твердого раствора, имеющих размер $L=128 a$. Для изучения поздней стадии распада выполнялась одна попытка расчетов для каждого из составов $c_{M}$ и параметров $\Delta$ с шагом по времени, равным $\Delta \tau=10^{-3}$. Рассмотрение основных характеристик второй фазы на ранней стадии распада $(\tau \leq 25)$ проводилось как результат усреднения по четырем независимым расчетам с различными реализациями начальных условий, при этом шаг по времени считался равным $\Delta \tau=0.5 \cdot 10^{-3}$.

Сравнение результатов двух расчетов с разным шагом $\Delta \tau$ в один и тот же момент времени $\tau$ позволило также оценить ошибку вычисления концентрационного поля, связанную с реализацией разностной схемы (5) (см. [24]). Оценка данной ошибки для концентрационного поля $c(x, y, z)$ составила $\sim 3 \cdot 10^{-5}$. Относительная ошибка определения среднего размера преципитата составила $\sim 10^{-4}$.

\section{5. Результаты моделирования}

На основе разработанного подхода было проведено моделирование распада твердых растворов для трех различных составов сплава для случаев наличия и отсутствия границы зерна (см. п. 4).

Как показывают результаты моделирования, для составов $c_{M}=14$ и 16 at.\% образование преципитатов происходило по конкурентному механизму, когда формирование преципитатов наблюдается как вблизи границ, так и в объеме зерна. Пример эволюции распределения фаз приведен на рис. 1 для сплава, имеющего состав $c_{M}=14$ at.\%. Для рассматриваемого положительного значения $\Delta>0$ зарождение и рост второй фазы происходит на участках вблизи границы зерна $(\sim 10 a)$, а также в его объеме. Непосредственно на границе зерна образование преципитатов не происходит, поскольку при $\Delta>0$ это привело бы к повышению энергии рассматриваемого фрагмента твердого раствора.

Для состава $c_{M}=12$ at.\% имела место преимущественная преципитация вблизи границы зерна, при которой выделения располагаются на некотором расстоянии от границы $(\sim 10 a)$, а в объеме полностью отсутствуют. Отсутствие преципитатов в объеме зерна означает, что равновесных флуктуаций оказывается недостаточно для образования зародышей критического размера. Таким образом, уменьшение концентрации растворенного компонента в сплаве приводит к существенному росту роли границ зерен и других дефектов в формировании зародышей критического размера. В отсутствие границы зерна для сплава $c_{M}=12$ at.\% преципитация не наблюдалась, что связано с существенным повышением работы зародышеобразования при уменьшении концентрации растворенного компонента [3].

На рис. 2-5 приведено сравнение кинетики характеристик второй фазы для всех рассматриваемых составов и параметров взаимодействия. Как следует из рис. 2-5, как при наличии границы зерна $(\Delta>0)$, так и при ее отсутствии $(\Delta=0)$ в процессе распада наблюдаются различные стадии: зарождение, диффузионный рост и коалесценция. Между диффузионной стадией и стадией коалесценции может наблюдаться также промежуточная стадия, характеризуемая достаточно медленным степенным ростом среднего размера преципитатов, что связано с трансформацией функции распределения по размерам $[19,21]$.

В случае конкурентной преципитации $\left(c_{M}=14\right.$, 16 at.\%) на начальном этапе образуется значительное количество зародышей второй фазы вблизи границы зерна (рис. 1, $a, 2,3$ ), затем, с заметной задержкой, процесс зарождения происходит вдали от границы зерна (рис. 1, $b, 2,3)$. Высокая концентрация преципитатов вблизи границы приводит к быстрому истощению зернограничной области по атомам растворенного компонента, поэтому их средний размер оказывается заметно меньше, чем в объеме зерна (рис. $1, c, d$ ). В связи с этим на поздней стадии распада, когда основным механизмом является коалесценция, образовавшиеся вблизи границ зерен преципитаты имеют тенденцию к растворению (рис. $1, c, d)$. В результате можно ожидать, что вблизи границы зерна может образоваться достаточно широкая 

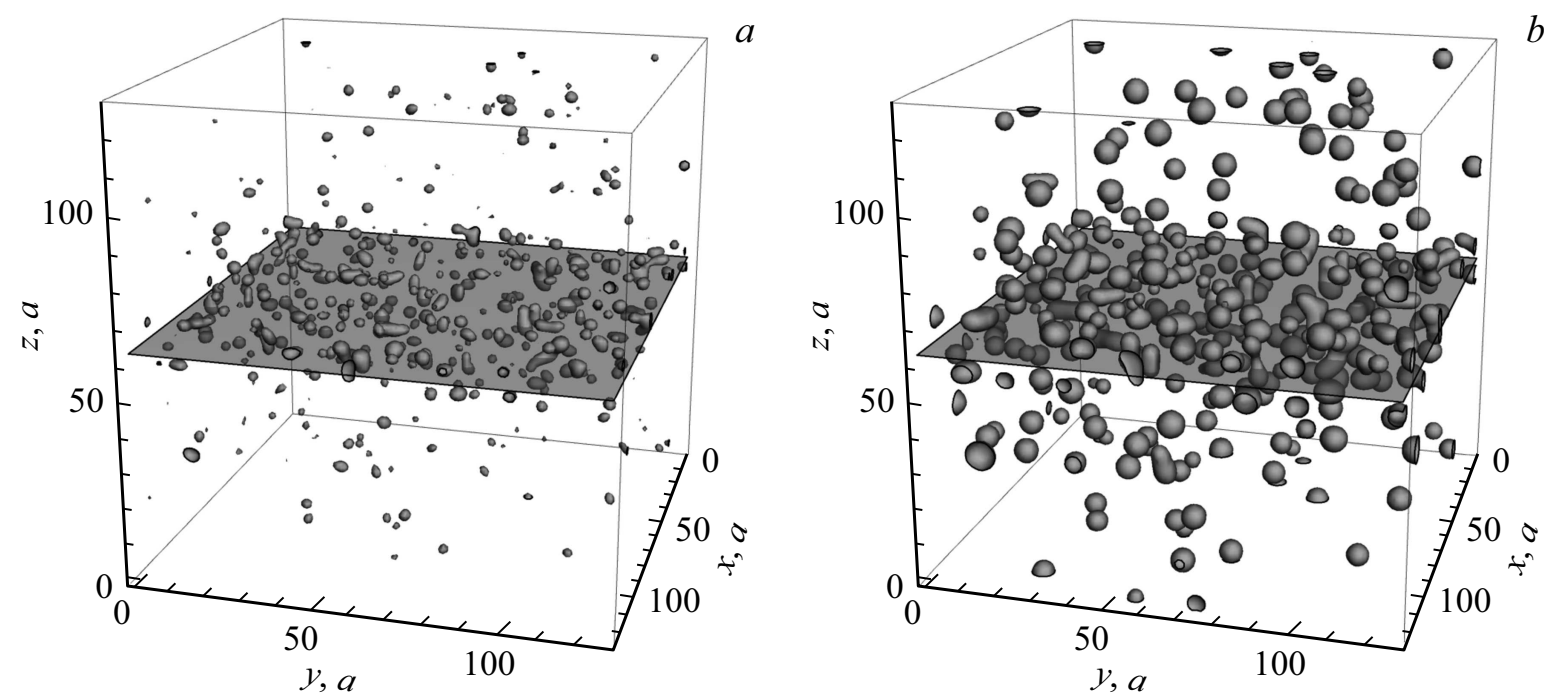

$b$
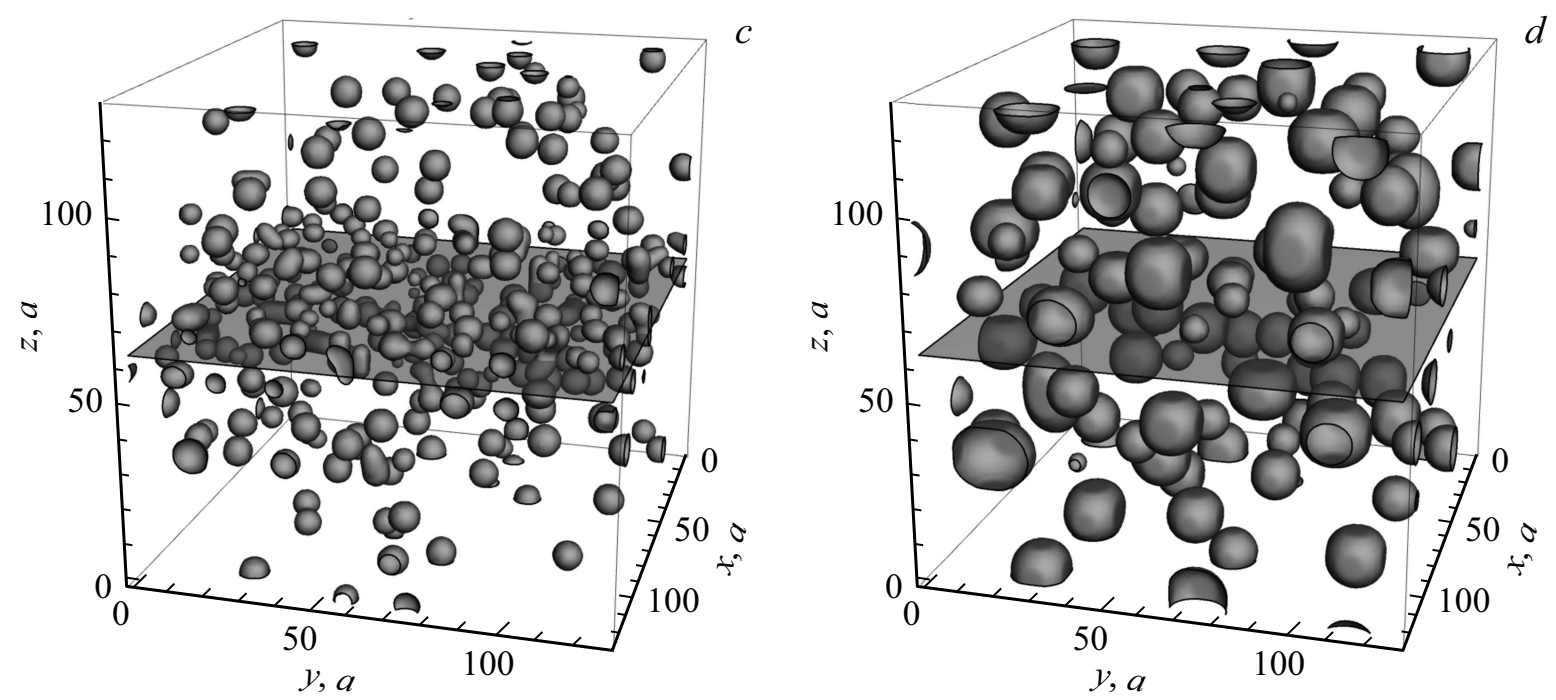

Рис. 1. Распределение фаз в бинарном сплаве, содержащем границу зерна $\left(c_{M}=14\right.$ at. $\left.\%, T^{*}=0.65, \Delta=0.5\right)$ в различные моменты времени: $a-\tau=5, b-\tau=35, c-\tau=55, d-\tau=1435$. Расположение границы зерна выделено плоскостью $(z=L / 2)$.

область (в несколько раз превышающая величину $\delta$ ), в которой преципитаты полностью отсутствуют. Образование протяженных зон, характеризуемых малой концентрацией растворенного компонента, а также отсутствием выделений второй фазы, наблюдается экспериментально в целом ряде сплавов на основе алюминия [24,25].

Как следует из рис. 3 и 4, уменьшение концентрации растворенного компонента приводит к уменьшению скорости зародышеобразования как в объеме зерна, так и вблизи границы. Если для монокристалла $(\Delta=0)$ скорость зародышеобразования в рассматриваемом случае снизилась почти на порядок при уменьшении состава от 16 до 14 at.\% (кривые 2 на рис. 3 и 4), то для границы зерна уменьшение составило примерно в два раза при изменении концентрации от 16 до 12 at.\% (рис. 3, кривые 4,3, рис. 4 кривая 3). Необходимо отметить, что наличие границы зерна привело к суще- ственному (почти на порядок) уменьшению инкубационного времени формирования зародышей второй фазы (рис. 3 и 4).

Одной из важнейших задач теории фазовых переходов является определение асимптотических участков на зависимостях среднего размера и концентрации от времени. Данные асимптотические участки обычно связываются с протеканием различных стадий распада, обусловленных действием разных механизмов [3,17-20,26], что имеет важное значение для прогнозирования распределения фаз и изменения различных свойств сплавов с течением времени. Для стадии диффузионного роста наблюдается увеличение среднего размера преципитатов в зависимости от времени по степенному закону $\langle R\rangle \propto \tau^{1 / 2}$ при слабом изменении их концентрации [3]. На стадии коалесценции $[3,26]$ средний размер преципитатов увеличивается по степенному закону $\langle R\rangle \propto \tau^{1 / 3}$ при убывании их концентрации $X_{C} \propto \tau^{-1}$. 
Промежуточная стадия [19,21], которая может наблюдаться между стадиями диффузионного роста и коалесценции, характеризуется существенно замедленным степенным изменением среднего размера $\langle R\rangle \propto \tau^{\alpha}$ и концентрации $X_{C} \propto \tau^{\beta}(0<\alpha<1 / 3,-1<\beta<0)$ преципитатов.

На рис. 5 проведен сравнительный анализ участков, на которых зависимости среднего радиуса преципитатов от времени описываются степенными функциями для случаев $\Delta=0$ и $\Delta=0.5$. Данный анализ позволяет установить ряд особенностей распределения фаз,

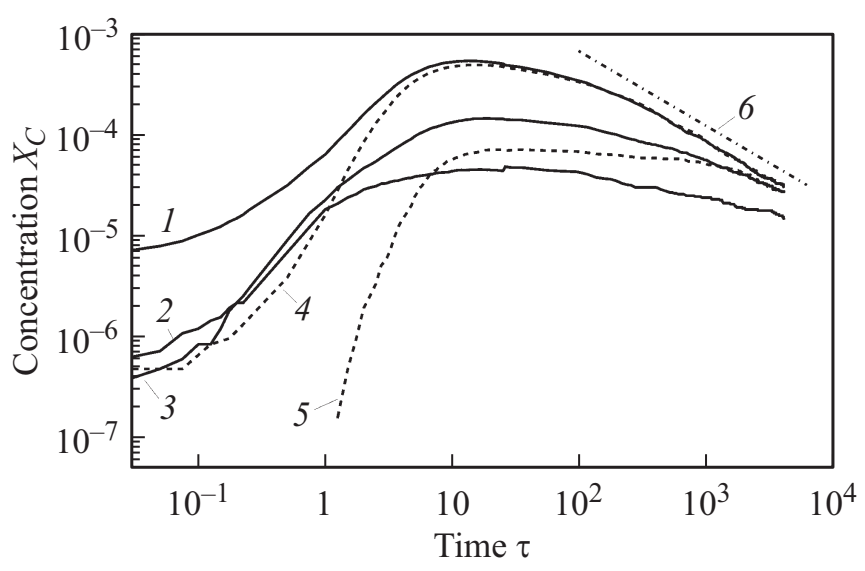

Рис. 2. Зависимость концентрации преципитатов от времени отжига в бинарных сплавах при температуре $T^{*}=0.65$ для различных составов: $1-c_{M}=12$ at.\%, $\Delta=0.5$, $2-c_{M}=14$ at. $\%, \quad \Delta=0.5,3-c_{M}=16$ at.\%, $\Delta=0.5$, $4-c_{M}=14$ at. $\%, \Delta=0,5-c_{M}=16$ at.\%, $\Delta=0$. Штрихпунктирная линия 6 соответствует аппроксимации на поздней стадии кривых 3 и 5 с помощью степенной функции $X_{C} \propto \tau^{-0.73 \pm 0.01}$

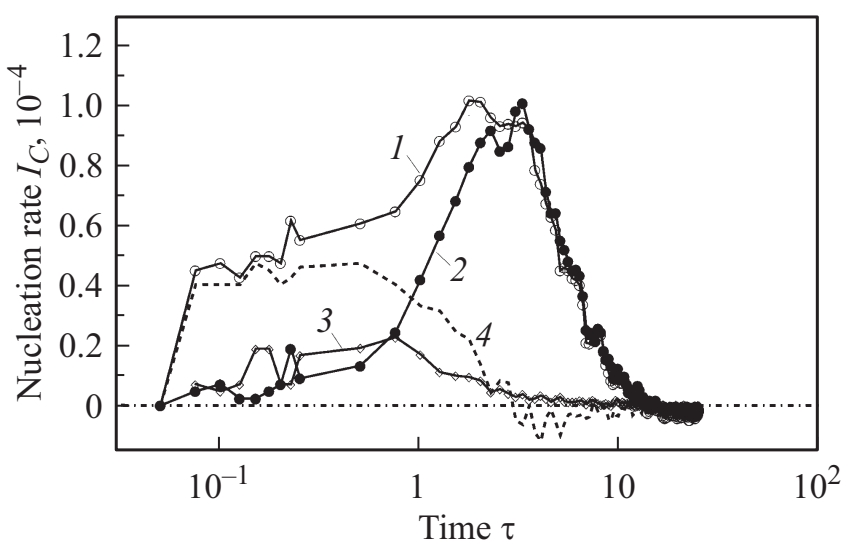

Рис. 3. Зависимость скорости нуклеации от времени отжига для бинарных сплавов при температуре $T^{*}=0.65$. Сплошными линиями отражены скорости нуклеации для различных составов и параметров взаимодействия $\Delta: 1-c_{M}=16$ at.\%, $\Delta=0.5,2-c_{M}=16$ at. $\%, \Delta=0,3-c_{M}=12$ at. $\%, \Delta=0.5$. Пунктирная линия 4 соответствует скорости зернограничной преципитации (получена как разность кривых 1 и 2) для сплава, характеризуемого составом $c_{M}=16$ at.\% и параметром $\Delta=0.5$.

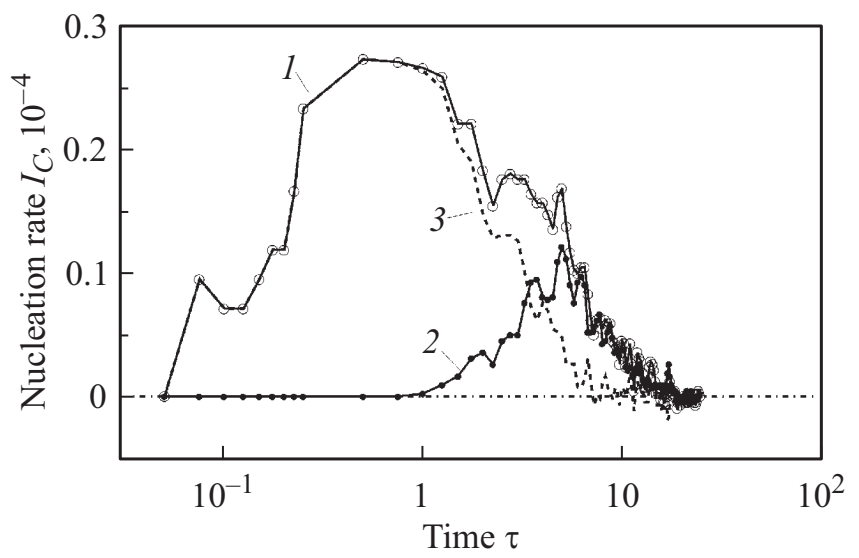

Рис. 4. Зависимость скорости нуклеации от времени отжига для бинарных сплавов при температуре $T^{*}=0.65$. Сплошными линиями отражены скорости нуклеации для различных составов и параметров взаимодействия $\Delta: 1-c_{M}=14$ at.\%, $\Delta=0.5,2-c_{M}=14$ at. $\%, \Delta=0$. Пунктирная линия 3 соответствует скорости зернограничной преципитации (получена как разность кривых 1 и 2 ).

обусловленных влиянием границ зерен на различных стадиях. Как следует из рис. 5, наличие границ зерен приводит к возникновению слабого степенного роста среднего размера преципитатов на ранней стадии распада. Данная особенность становится более выраженной при уменьшении состава сплава (рис. 5, a, c,e). Для сплава с бо́льшим значением состава $\left(c_{M}=16\right.$ at.\%) или в отсутствие границ зерен данный участок выражен слабо (рис. $5, a, b, d)$.

На стадии диффузионного роста наличие границы зерна приводит к заметному замедлению скорости роста $\left(\langle R\rangle \propto \tau^{\alpha}\right)$. В отсутствие границы зерна показатель степени $\alpha$ оказывается равным $\alpha=0.45$ для состава $c_{M}=16$ at.\% и $\alpha=0.53$ для состава $c_{M}=14$ at.\%. Данные значения показателя оказываются достаточно близкими к выводам теории диффузионного роста, в которой установлено $\alpha=1 / 2$. При наличии границы зерна значение показателя $\alpha$ оказывается существенно ниже для всех трех рассмотренных сплавов и демонстрирует очень близкие друг к другу значения $\alpha=0.40 \pm 0.01$. Существенное понижение скорости роста может быть связано с рядом факторов. Во-первых, вблизи границы зерна, ввиду высокой концентрации преципитатов, имеет место их слияние (коагуляция), что может приводить к снижению показателя степени $\alpha$ [19,21]. Во-вторых, распад сплава может протекать по смешанному механизму, когда в зернограничной области и в объеме зерна могут доминировать разные процессы (зарождение, диффузионный рост, коагуляция). В-третьих, имеет место анизотропия потоков компонентов раствора, связанная с наличием границы зерна, которая представляет собой (в случае $\Delta>0$ ) потенциальный барьер для атомов растворенного компонента. 

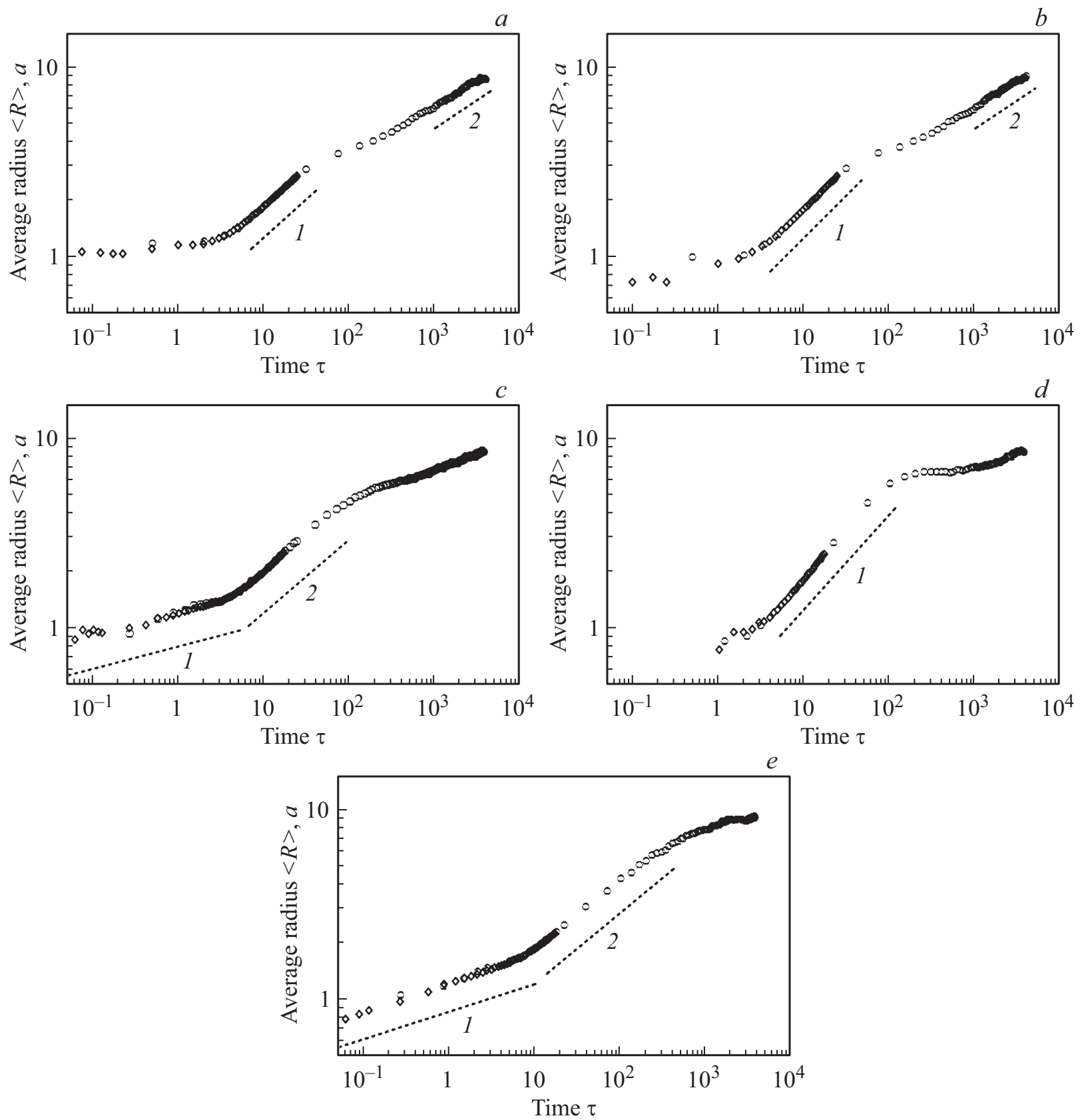

Рис. 5. Зависимость среднего радиуса преципитатов от времени отжига в бинарных сплавах при температуре $T^{*}=0.65$ для различных составов: $a-c_{M}=16$ at. $\%, \Delta=0.5, b-c_{M}=16$ at.\%, $\Delta=0, c-c_{M}=14$ at.\%, $\Delta=0.5, d-c_{M}=14$ at. $\%$, $\Delta=0, e-c_{M}=12$ at. $\%, \Delta=0.5$. Пунктирные линии соответствуют участкам степенного роста $\langle R\rangle \propto \tau^{\alpha}$ (рис. $a: 1-\alpha=0.41$, $2-\alpha=0.30$; pис. $b: 1-\alpha=0.45,2-\alpha=0.30$; рис. $c: 1-\alpha=0.13,2-\alpha=0.41$; рис. $d: 1-\alpha=0.53$; pис. $e: 1-\alpha=0.15$, $2-\alpha=0.40)$. Точки получены при решении уравнения Кана-Хилларда: ромбы - результат расчета при $\Delta \tau=0.5 \cdot 10^{-3}$ и усреднения по четырем независимым попыткам, круги - результат расчета при $\Delta \tau=10^{-3}$ для одной попытки.

Для рассмотренных параметров взаимодействия вблизи границ зерен $\Delta$ и составов сплава $c_{M}$ переход к выраженной стадии коалесценции наблюдался только для сплавов, характеризуемых концентрацией $c_{M}=16$ at.\%. В данном случае влияние границ зерен на распределение компонентов твердого раствора оказывается крайне слабым (см. рис. 5, $a, b$ ), так как основные процессы в данном случае протекают вдали от границ зерен.
Уменьшение состава сплава или увеличение температуры приводит к существенному удлинению продолжительности диффузионной и промежуточной стадий распада (см. также $[19,21])$. Поэтому для случаев меньших концентраций наблюдалась только промежуточная стадия распада, являющаяся переходной между стадиями диффузионного роста и коалесценции $[19,21]$. Промежуточная стадия в присутствии границ зерен харак- 
теризуется медленным степенным ростом (рис. 5,c), в то время как для монокристаллического фрагмента твердого раствора (рис. 5,d) средний размер преципитатов практически не изменяется. Для наименьшего из рассмотренных составов $c_{M}=12$ at.\% наблюдались только стадии зарождения, роста, а также промежуточная стадия. Более подробное изучение поздней стадии распада, протекающей по механизму коалесценции, с учетом границ зерен требует рассмотрения фрагментов твердого раствора заметно большего размера и более длительных интервалов времени, что приводит к существенному повышению (по крайней мере на два порядка) затрат времени на моделирование.

\section{6. Заключение}

В данной работе рассмотрено влияние границ зерен на кинетику преципитации в бинарных сплавах. В качестве основного допущения модели используется предположение об изменении параметров взаимодействия между компонентами твердого раствора по мере приближения к границам зерен. В рассматриваемом в данной работе случае считается, что эффективная энергия смешения вблизи границы зерна увеличивается, что может быть, например, обусловлено вкладом поля упругих напряжений.

Проведенное моделирование позволило установить некоторые особенности влияния границ зерен на формирование выделений второй фазы вблизи границы зерна:

1) Если концентрация растворенного компонента велика, имеет место конкурентная преципитация, при которой образование выделений второй фазы происходит как вблизи границ зерен, так и в объеме зерна. Зародыши, образовавшиеся вблизи границы зерна, характеризуются большей концентрацией и меньшим размером, чем в объеме зерна. В результате на поздней стадии данные преципитаты имеют тенденцию к растворению, а вблизи границы зерна образуется достаточно широкая обедненная область, в которой концентрация растворенного компонента мала и отсутствуют выделения второй фазы.

2) При малых концентрациях растворенного компонента происходит зародышеобразование только вблизи границ зерен, а в объеме данный процесс не происходит ввиду недостаточности равновесных флуктуаций для преодоления барьера зародышеобразования. По всей видимости, данная особенность должна распространяться и на другие дефекты микроструктуры (дислокации, дисперсные частицы и др.), вблизи которых может происходить повышение эффективного параметра взаимодействия между компонентами твердого раствора.

3) Наличие границ зерен может приводить к изменению скорости роста среднего размера преципитатов $\langle R\rangle \propto \tau^{\alpha}$. Во-первых, при малых концентрациях растворенного компонента на ранней стадии распада наблюдался выраженный участок медленного степенного роста
( $\alpha \sim 0.13-0.15)$. В отсутствие границ зерен, а также для бо́льших значений концентрации данный участок обнаружен не был. Во-вторых, на стадии диффузионного роста наличие границ зерен также приводит к существенному уменьшению скорости роста среднего размера $(\alpha \sim 0.41)$. Замедление скорости роста на данных участках, по всей видимости, связано с анизотропией диффузионных потоков, обусловленной наличием границы зерна, а также сочетанием различных механизмов распада (например, зарождения, диффузионного роста и коагуляции).

На поздней стадии распада скорость роста для бо́льших значений составов сплава слабо зависит от наличия границы зерна и удовлетворительно описывается степенной зависимостью с $\alpha \sim 0.3$, что оказывается достаточно близким к классической теории коалесценции. Для меньших значений состава сплава влияние границ зерен на скорость роста на поздней стадии установить не удалось, ввиду необходимости существенного (на два порядка) увеличения вычислительных ресурсов или времени моделирования.

\section{Список литературы}

[1] L. Priester. Grain Boundaries: From Theory to Engineering. Dordrecht: Springer. (2013). 441 p.

[2] В.Н. Чувильдеев. Неравновесные границы зерен в металлах. Теория и приложения. Физматлит М. (2004). 304 с.

[3] K. Kelton, A. Greer. Nucleation in Condensed Matter. Elsevier. (2010). 726 p.

[4] S.E. Restrepo, S.T. Giraldo, B.J. Thijsse. Mod. Simul. Mater. Sci. Eng. 21, 055017 (2013).

[5] P. Lejcek, S. Hofmann, J. Janovec. Mater. Sci. Eng. A 462, 76 (2007).

[6] A. Kuronen, S. Granroth, M.H. Heinonen, R.E. Perälä, T. Kilpi, P. Laukkanen, J. Lang, J. Dahl, M.P.J. Punkkinen, K. Kokko, M. Ropo, B. Johansson, L. Vitos. Phys. Rev. B 92, 214113 (2015).

[7] С. Клоцман. УФН 160, 99 (1990).

[8] В.В. Слезов, Л.Н. Давыдов, В.В. Рогожкин. ФТТ. 37, 3565 (1995).

[9] M.K. Mitra, M. Muthukumar. J. Chem. Phys. 134, 044901 (2011).

[10] П.Е. Львов, В.В. Светухин. ФТТ 59, 2425 (2017).

[11] J.W. Cahn, J.E. Hilliard. J. Chem. Phys. 28 (1958) 258.

[12] J. Cahn. Acta Met. 9, 795 (1961).

[13] A. Umantsev. Field theoretic method in phase transformations. Springer. N.Y. (2012). 343 p.

[14] I. Steinbach. Mod. Simul. Mater. Sci. Eng. 17, 073001 (2009).

[15] N. Provatas, K. Elder. Phase-field methods in material science and engineering. Wiley-WCH, Weinheim. (2010). 298 p.

[16] L. Vanherpe, N. Moelans, B. Blanpain, S. Vandewalle. Phys. Rev. E 76, 056702 (2007).

[17] L.-Q. Chen, J. Shen. Comput. Phys. Commun. 108, 147 (1998).

[18] J. Zhu, L.-Q. Chen, J. Shen, V. Takare. Phys. Rev. E 60, 3564 (1999). 
[19] П.Е. Львов, В.В. Светухин. ФТТ 59, 345 (2017).

[20] M. Miller, R. Forbes. Atom-probe tomography: the local electrode atom probe. Springer, N.Y. (2014). 423 p.

[21] P.E. L'vov, V.V. Svetukhin. Mod. Simul. Mater. Sci. Eng. 25, 075006 (2017).

[22] П.Е. Львов, В.В. Светухин. Свидетельство о государственной регистрации программы для ЭВМ. № 2017616554 от 08.07.2017.

[23] S. Dai, Q. Du. J. Comp. Phys. 310, 85 (2016).

[24] D.A. Porter, K.E. Easterling. Phase transformations in metals and alloys. 2nd ed. Springer, Hong-Kong. (1992). 514 p.

[25] S. Hirosawa, Y. Oguri, T. Sato. Mater. Trans. 46, 1230 (2005).

[26] В.В. Слезов, В.В. Сагалович. УФН 151, 67 (1987). 